\title{
La valeur de 1" "impersonnel" dans Un barrage contre le Pacifique de Marguerite Duras: Analyse sémiotique du discours littéraire
}

\section{The Impersonal in A Dam Against the Pacific by Marguerite Duras: Semiotic Analysis of the Literary Discourse}

\author{
Nedret KILIÇERI' ${ }^{1}$
}

${ }^{1}$ Prof. Dr., Istanbul University, Faculty of Letters, Department of Western Languages and Literature, French Language and Literature Department, Istanbul, Turkey

ORCID: N.K. 0000-0003-3445-1275

Corresponding author:

Nedret KILIÇERI,

Istanbul University, Faculty of Letters, Department of Western Languages and Literature, French Language and Literature Department, Istanbul, Turkey

E-mail: nedretoztokat@yahoo.fr

Submitted: 19.02 .2020

Revision Requested: 28.03.2020

Last Revision Received: 30.03.2020

Accepted: 01.04.2020

Citation: Kiliceri, N. (2020). La valeur de I'"impersonnel" dans Un barrage contre le Pacifique de Marguerite Duras: Analyse sémiotique du discours littéraire. Litera, 30(1), 1-25.

https://doi.org/10.26650/LITERA2020-0047

\section{RÉSUMÉ}

Un barrage contre le Pacifique est l'un des romans de Marguerite Duras qui puise sa source dans la vie de l'auteure. L'histoire racontée trace le panorama succinct de I'Indochine des années 1940. Le roman traite d'une époque où des milliers de familles françaises sont parties pour l'Indochine pour une meilleure vie. L'une de ces familles est celle des Duras. Cet article vise une analyse sémiotique d'un extrait du roman (Duras, 1950, p. 115-119) qui est une description de la plaine où vivent les protagonistes et les indigènes. La longue description de la plaine de Kam qui est notre corpus d'analyse dessine non seulement un espace topographique précis, mais aussi l'espace de vie des indigènes. Notre analyse s'attarde sur les éléments récurrents du discours du narrateur pour dévoiler l'organisation sémantique proposé par l'univers général du récit. Notre approche est celle de l'analyse sémiotique du discours littéraire telle qu'elle est élaborée et pratiquée dans les travaux d'A. J. Greimas, de D. Bertrand de J. Fontanille, mais aussi par les linguistes et narratologues J.-M. Adam et J.-P. Goldenstein. L'analyse prend en considération les deux aspects du texte : l'énoncé et l'énonciation. Les unités discursives fréquemment employés dans cette description dessinent les isotopies du corpus. Les éléments figuratifs et thématiques permettent d'aborder l'organisation profonde de la signification du texte.

Mots-clés: Analyse sémiotique, discours littéraire, description, figure, isotopie

\section{ABSTRACT}

A dam against the Pacific is one of Marguerite Duras's novels and is based on the author's life. The story told traces the succinct panorama of Indochina in the 1940s. The novel is about a time when thousands of French families were leaving for Indochina for a better life. One of these families is the Duras family. This article is based on a semiotic analysis of an excerpt from the novel (Duras, 1950, p. 115-119) which is a description of the plain where the protagonists and the natives live. The long description of the Kam Plain, which is our corpus of analysis, draws not only a precise topographical space, but also the living space of the natives. Our analysis focuses on the recurring elements of the narrator's discourse to reveal the semantic organization proposed by the general universe of the narrative. Our approach is 
that of semiotic analysis of literary discourse as it is elaborated and practiced in the work of A. J. Greimas, D. Bertrand and J. Fontanille, but also by linguists and narratologists J.-M. Adam and J.-P. Goldenstein. The analysis takes into account two aspects of the text: the statement and the enunciation. The discursive units frequently used in this description draw the isotopies of the corpus. The figurative and thematic elements allow us to address the profound organization of the meaning of the text.

Keywords: Semiotic analysis, literary discourse, description, figure, isotopy

\section{EXTENDED ABSTRACT}

Duras's novel, with its richness and depth, lends itself to a plurality of readings. The novelistic discourse takes its strength by the evocation of the misery of the country, the mother's effort to survive with her uncultivable plantation, the injustices of the colonial system, and by her intimate and distant writing that describes eminently fate and the misery of the natives and the «little whites» of the country.

In the first part of the novel, we will dwell on a fragment of the novel (Duras, 1950, p. 115-119) where the narrative focuses the Kam plain through a detailed description whose imaged style effectively shows the miserable conditions which the natives are subjected to. The extract highlights a poor landscape of Indochinese flora populated by children who are in a way the linchpin of the description. Around them are described the parents of these children and finally another group that surrounds them, namely the stray dogs of the plain.

This way of presenting collective actors (in three interrelational circles) not only shows the unfavourable aspect of this space, but it also shows in the menus the shortage, hunger, and contagious diseases that reign in the country. The long description draws a topographical space, which at the same time appears as a thymic space since it is linked to the harsh ordeal of the Mother culminating in the failure from which she will never recover. Thus, the mother's effort to cultivate rice on this uncultivable land (which gives the title to the novel, since it builds a dam against the Pacific) appears as the culmination of the thematic network of the novel. Just as nothing can be said against the indomitable tides of the ocean, here any survival effort is also doomed to failure.

This analysis is essentially based on the semiotic approach to literary discourse, which relies on narrative processes as a constituent element of the narrative. It should be remembered that the literary text consists of two levels, one of which concerns the 
semantics of the content and the other, the manifestation of meaning. According to this approach, the literary text organizes the messages in an interrelation way to give rise to the discourse. It is therefore necessary to take into account the units of the discursive manifestation (lexemes) and the componential units of meaning associated with it.

The corpus of our analysis illustrates the thematic and figurative axes of the novel and appears as a significant example of the novelistic discourse that emphasizes the anonymous characters of the story told, namely the natives. Concerning the observer point of view, the children appear as an anonymous and collective figure whose tragic fate is combined with that of the small settlers of the country.

In the text, children are compared to animals and plants that grow in the plain naturally. Their lives and survival, like death, are an ordinary fact, so much so that their birth and death are invariably subject to the indifference of their families. Comparative processes, the use of verbs and adjectives illustrate the point of view chosen in the narrative to describe the shabby conditions of the plain as a space of the lives of the natives.

The discourse of stylistic processes bringing «children» and «dogs» together in cataclysm, life and death in indifference is conveyed in a particular way by a network of anaphoras used mainly for «children». Analysis of the frequency of «indefinites» and their semi-Semitic content showed the approximation or even erasure of the semantic categories «/animal/human,» «living/dead,» «sense/insignificance» in the thematic configuration defining «children.»

Thus, the large number of indefinite pronouns with an indefinite value affects the thematic isotopy «inanity» defined as «character of what is empty, without real content, which has no interest to the heart or the mind» to describe the being of children of the plain. The entire anaphoric system of the corpus emphasizes the non-meaning nature of children by reducing them in the discourse to the «inanimate» state.

As a result, the semantic line between «animated/inanimate,» between «human/ animal» and «sensible/insignificant» fades into the text. We can thus consider the game of inversion and/or approximation of the semantic values of the figures as «children, dogs, life, death, scourge» as a fact of style by playing on the referential ambiguity of these figures in relation to their figurative and thematic content. 
As in this analyzed excerpt, Duras's novel is in itself a denunciation of the unworthy conditions inflicted not only on the French expatriates in the colonies, but also on the peoples of these countries. The new life that the Mother wanted to build here has become a perfect example of failure (debt, poverty, boredom, distress, despair). But as the description of life on the plain, in these confines, shows us, there is no effective way to reorganize a prosperous life. The excerpt shows, through the strong images, the vain determination of the Mother to build the dams against the Pacific. 


\section{Introduction}

Un barrage contre le Pacifique de Marguerite Duras s'inspire de la vie de son auteure. Il dessine derrière l'histoire d'une famille française, un panorama succinct de l'Indochine des années 1940. Le roman traite d'une époque où des milliers de familles françaises sont parties pour l'Indochine pour une meilleure vie. L'une de ces familles est celle des Duras.

Marguerite Duras est née en Indochine le 4 avril 1914 à Gia Dinh, près de Saigon. Son père a enseigné les mathématiques et sa mère était institutrice. Son père rentre à Paris pour des raisons de santé en 1926 où il meurt quelques années plus tard. Sa mère continue à enseigner dans les écoles indigènes de la colonie au Cambodge et à Sadec. La famille mène une vie modeste loin des promesses de richesse et de prospérité exaltées par le gouvernement français colonialiste. Marguerite et ses deux frères grandissent avec les enfants indigènes. La jeune fille quitte le foyer en 1932 pour suivre ses études en France.

Le roman reprend certains éléments autobiographiques de la vie de $M$. Duras, en l'occurrence le portrait de la mère institutrice, l'absence du père directeur d'école évoqué dans la narration et le milieu où la famille vit en sont les plus prépondérants.

Un barrage contre le Pacifique invite son lecteur à l'exploration d'un univers romanesque conçu au croisement du réel et de l'imaginaire, et à une écriture exemplaire témoignant de mode de vie et de la manière d'être des personnages principaux et secondaires. Commencé en 1947 et publié en 1950, le roman manque de peu le Prix Goncourt ; néanmoins il assurera à Duras la place qu'elle méritait grâce à son écriture singulière.

L'intrigue du roman est construite autour d'une petite famille française installée en Indochine en rêvant d'une meilleure vie comme le prônait le mouvement colonialiste. La mère qui est une ancienne institutrice essaie de vivre avec ses deux enfants Joseph et Suzanne sur un terrain incultivable qu'elle a loué sans le savoir pour mener une vie prospère.

Comme la plantation ne les aide pas de vivre honnêtement, tous les trois cherchent des moyens pour assurer leur survie : Joseph rêve d'assurer le transport dans la plaine de Kam, ou de vendre le phonogramme qu'il aime tant ; la mère tente de rentabiliser son domaine grâce à un barrage voué à l'échec contre l'océan ; Suzanne essaie d'obtenir 
les faveurs d'un jeune et riche rentier... Autant d'efforts qui traduisent le grand désespoir de la famille au sein de l'Indochine française partagée entre le pouvoir, les grands colons et les "petits blancs" aussi malheureux que les indigènes. Contrairement à la Mère qui a su supporter cette misère imposée par le système colonisateur, Joseph et Suzanne dont l'unique envie a toujours été de s'évader de la plaine refuseront d'y rester et la quitteront après la mort de la Mère.

Par la richesse et la profondeur de sa thématique, le roman de Duras se prête à une pluralité de lectures. Le discours romanesque prend sa force par l'évocation de la misère du pays, l'effort de la mère pour subsister avec sa plantation incultivable, les injustices du système colonial, et aussi par son écriture intime et distante à la fois qui décrit éminemment le destin et la misère des indigènes et des "petits blancs" du pays.

Dans la première partie du roman, nous nous attarderons sur un fragment du roman (Duras, 1950, p. 115-119) où la narration focalise la plaine de Kam à travers une description détaillée dont le style imagé montre avec efficacité les conditions misérables auxquelles sont soumis les indigènes. L'extrait met en exergue le paysage indochinois peuplé d'enfants qui constituent en quelque sorte le pivot de la description. Autour d'eux, sont décrits les parents de ces enfants; et enfin un autre groupe qui les entoure, à savoir les chiens errants de la plaine.

Cette manière de présenter les acteurs collectifs (en trois cercles interrelationnels), ne montre pas seulement l'aspect défavorable de cet espace, mais elle décrit dans les menus détails les atrocités de la misère qui règne dans le pays. La longue description dessine l'espace topographique de I'histoire racontée, qui apparaît en même temps comme un espace thymique, puisqu'il illustre la dure épreuve de la Mère aboutissant à un échec dont elle ne se remettra jamais. Ainsi l'effort de la Mère, pour cultiver le riz sur ce terrain incultivable, (qui donne le titre au roman) apparaît-il comme le point d'orgue dans le réseau thématique du roman. De même qu'on ne peut rien contre les marées indomptables de l'océan, dans une telle contrée, tout effort de survie est également condamné à l'échec. Or c'est à ce même océan Pacifique qui borde ces terrains arides que la mère « avait rapporté ses rêves »(Duras, 1950, p. 33).

L'extrait du roman sur lequel se base notre analyse représente l'absence de toute valeur affective et économique dans la plaine marécageuse de Kam. A la page 33 du roman, il est noté que cette plaine entourée de l'océan Pacifique et d'une longue chaîne 
de montagne qui l'enferme vers l'Est comme un mur, se joint à l'océan suivant une courbe qui descend jusqu'au golfe de Siam pour devenir de petites îles vêtues des sombres forêts tropicales. Il s'agit d'un terrain presqu'ennemi de l'agriculture, défavorable pour la prospérité sociale et économique, qui construit, au niveau narratif, le cadre général du roman.

\section{Corpus d'analyse et approche du texte}

L'extrait de notre analyse se compose de la description de la plaine de Kam où se trouve le bungalow de la Mère et de ses enfants, Joseph et Suzanne. Le passage se situe entre les pages 115 et 119 de la première partie du roman ; il expose en détails leur vie commune à tous les trois. Après avoir perdu leur cheval qu'ils avaient acheté pour gagner de l'argent en faisant le transport dans la plaine, déprimés ils partent pour se distraire au village à Ram où ils rencontrent un certain M. Jo. Séduit par Suzanne, il vient leur rendre visite. La présence de ce riche exploiteur disperse leur ennui en provoquant chez eux un bizarre sentiment d'espoir de se débarrasser de leurs dettes malgré leur hésitation morale devant les avances faites par M. Jo à Suzanne. La suite du roman montrera leur effort pour changer leur vie, et ceci, jusqu'à la mort de la Mère.

La description nous montre l'espace topographique où cette famille française est obligée de vivre. Cet espace se présente à travers les acteurs qui le peuplent et les conditions dures de la vie ; il apparaît ainsi comme l'horizon de la vie des trois protagonistes dans ce coin de l'Indochine.

En rappelant à la suite de Rastier que « l'ensemble des propositions dans un texte par un acteur qui émet des signes, linguistiques ou non, constitue l'univers de cet acteur, quelles que soient la valeur de vérité de ces propositions et la nature de leurs arguments » (1989, p. 161), la plaine de Kam s'avère comme l'espace englobant l'être et le faire de la Mère, de Joseph et de Suzanne.

L'extrait que nous avons choisi montre de façon significative la relation de la Mère avec ce pays. En dehors de ses soucis pour régler ses dettes pour la concession, deux préoccupations la retiennent sur ce territoire, à savoir les plantes et les enfants indigènes: «Quand ce n'était pas aux plantes, c'est aux enfants que la mère s'intéressait » (Duras, 1950, p. 115). 
Cette analyse s'inspire essentiellement de l'approche sémiotique du discours littéraire qui s'appuie sur les procédés narratifs comme éléments constituant le récit. Rappelons que le texte littéraire se compose de deux niveaux dont l'un concerne la sémantique du contenu et l'autre, la manifestation de la signification (voir Greimas, 1966, p. 69). Dans un texte littéraire les messages s'organisent de façon interrelationnelle pour donner lieu au discours. Il convient ainsi de prendre en considération les unités de la manifestation discursive (lexèmes) et les unités de sens qui les sous-tendent.

La récurrence des unités sémantiques (la structure profonde de la signification) constitue les plans isotopes du discours qui assurent son homogénéité (Greimas, 1966, p. 71-72). Dans la sémiotique greimassienne, « un énoncé se présente d'abord comme un donné n'offrant que sa « surface » sous laquelle on peut trouver une organisation sous-jacente plus profonde » (Greimas et Courtés, 1993, p. 373). Le niveau des procédés de dénomination, d'organisations syntaxiques des unités discursives peut être considéré comme élément d'analyse pour aborder le niveau profond de la signification puisque «la structure de surface ne se définit que par rapport à la structure profonde » (Greimas et Courtés, 1993, p. 372). La structure profonde de la signification, elle concerne les instances de « génération de la signification » du discours qui organisent les structures complexes.

Au niveau profond, l'analyse cherche à repérer les isotopies car elles relèvent du niveau du contenu ; ce sont les plans sémantiques « internes au discours ». Une isotopie se construit par «l'itérativité des mêmes sèmes nucléaires ou classématiques à l'intérieur du discours » (Greimas et Courtés, 1993, p. 197-198).

Notre analyse s'attarde ainsi sur les éléments pertinents et répétitifs du niveau discursif pour essayer d'en dévoiler la charge sémantique afin de décrire les plans isotopes du texte. Les observations porteront ainsi sur l'énoncé-discours défini comme « toute grandeur pourvue de sens relevant de la chaîne parlée ou du texte écrit, antérieurement à toute analyse linguistique ou logique »; il est donc «l'état résultatif de l'énonciation » qui est essentiellement un acte -celui d'énoncer ou de produire un acte de langage (Greimas et Courtés, 1993, p. 123).

L'analyse de l'énoncé nous permettra de déterminer la position discursive du sujet de l'énonciation. D. Bertrand prend en considération «le point de vue comme une position, déterminé par le jeu des places énonciatives » dans le discours (2000, p. 75). 
Dans cet extrait descriptif, nous nous proposons donc d'observer les mécanismes qui régissent l'option du point de vue de l'énonciateur.

D'autre part, comme le propose J. Fontanille, le sujet de l'énonciation s'inscrit dans son énoncé-discours comme un sujet cognitif (1989, p. 40). L'observateur représente un contenu donné, et le contrôle. L'analyse de l'énonciation dans le texte narratif ne peut se passer des éléments textuels qui portent l'empreinte cognitive (justification, évaluation, information, etc.) de l'actant observateur. Telle sera notre vision sur l'acte narrateur mettant en scène les personnages de notre corpus.

Dans le cadre de la théorie de l'énonciation littéraire, nous partirons de l'énoncé pour y « retrouver les traces et les opérations de l'observateur, et les modes de construction des points de vue »; comme le souligne Fontanille, une telle démarche sert à «établir des parcours signifiants, des formes de contenu, par lesquelles les discours prédéterminent la participation de l'énonciataire à l'interprétation, et préparant en somme ses identifications » (Fontanille, 1989, p. 43).

Visant une analyse au niveau sémantique du contenu, nos observations porteront sur les possibilités d'une mise en corrélation du discursif (éléments énoncifs) et du niveau profond de la signification. Comme le propose, J. Courtés, la sémantique de l'énoncé (description du plan du contenu selon Hjelmslev) « rend compte -uniquement en ce qui concerne le seul signifié- de l'organisation d'un univers du discours » (1989, p. 9). Notre lecture cherchera à décrire la sémantique de l'énoncé dans ce qu'elle est représentée grâce à l'acte de l'énonciation.

Notre approche partira des éléments itératifs du discours énoncé; nous nous servirons du critère de fréquence discursive à partir du nombre de récurrences de certains termes du discours. Nos observations et constatations au niveau de l'énoncé nous permettront de mener l'analyse simultanément sur les deux plans du récit (à savoir les figures manifestes et les isotopies latentes) à partir de la description de la plaine de Kam fournie entre les pages 115 et 119 d'Un barrage contre le Pacifique. Elément fondamental d'analyse sémantique du contenu, « le concept d'isotopie s'est imposé en raison de la nature polysémique du contenu et de la nécessité, pour l'analyse du discours littéraire, d'opérer à un niveau transphrastique »; une définition récapitulative serait celle proposée par J.-M. Adam et J.-P. Goldenstein : «l'isotopie est un champ ouvert par la redondance d'unités linguistiques (manifestes ou non) du plan d'expression ou du plan du contenu » (1976, p. 97). 
Avant d'entamer notre lecture, nous avons recouru à la segmentation selon laquelle notre corpus se définit comme un ensemble discursif composé de cinq paragraphes, soit cinq séquences correspondant aux cinq étapes de l'analyse sémiotique. Comme l'analyse est basée sur l'emploi fréquent de l'acteur « enfants », nous avons souligné les anaphores et marqué en gras les substantifs pour enfants dans ces paragraphes situés au début de chaque analyse.

\section{Analyse par séquences}

\section{Séquence I : « II y avait beaucoup d'enfants dans la plaine »}

Il y avait beaucoup d'enfants dans la plaine. C'était une sorte de calamité. Il y en avait partout, perchés sur les arbres, sur les barrières, sur les buffles, qui, rêvaient, ou vautrés dans la vase à la recherche des crabes nains des rizières. Dans la rivière aussi on en trouvait qui pataugeaient, jouaient ou nageaient. Et à la pointe des jonques qui descendaient vers la grande mer, vers les îles vertes du Pacifique, il y en avait aussi qui souriaient, ravis, enfermés jusqu'au cou dans de grands paniers d'osier, qui souriaient mieux que personne n'a jamais souri au monde. Et toujours avant d'atteindre les villages du flanc de la montagne, avant même d'avoir aperçu les premiers manguiers, on rencontrait les premiers enfants des villages de forêts, tous enduits du safran contre les moustiques et suivis de leurs bandes de chiens errants. Car partout où ils allaient, les enfants trainaient derrière eux leurs compagnons, les chiens errants, efflanqués, galeux, voleurs de basses-cours, que les Malais chassaient à coups de pierre et qu'ils ne consentaient à manger qu'en période de grande famine, tant ils étaient maigres et coriaces. Seuls les enfants s'accommodaient de leur compagnie. Et eux n'auraient sans doute eu qu'à mourir s'ils n'avaient pas suivi ces enfants, dont les excréments étaient leur principale nourriture. (Duras, 1950, p. 115-116)

La séquence commence par cette constatation générale : « II y avait beaucoup d'enfants dans la plaine » à partir de laquelle tout un enchaînement discursif et thématique s'agence pour mettre en exergue l'aspect de la vie des indigènes. La métaphore «calamité » et la «Quantité » (Beaucoup) désignant les enfants introduisent dès cette première ligne de notre corpus, les effets de sens caractérisant l'être des enfants. 
Comme les quatre autres, la première séquence retrace l'espace principal, la « plaine » qui apparaît comme l'un des décors principaux du roman puisqu'elle dessine l'endroit où vivent la Mère, Suzanne et Joseph. Accompagnée des lexèmes « rivière », « les îles vertes de la Pacifique », « villages » et « la grande mer », « la plaine » dessine non seulement l'espace romanesque cher à l'auteure, mais aussi l'espace familier (Greimas, 1976, p. 97) des trois protagonistes du récit. Devenue la seconde patrie depuis une dizaine d'années, I'Indochine est leur territoire. Suzanne et Joseph se baignent dans le rac, partent à la chasse comme les autres habitants de la région.

Dans ce segment, l'espace de l'énoncé est lisible sur l'isotopie spatiale : la « plaine » est caractérisée dans l'énoncé par les sèmes nucléaires : /géographique/ + /vaste/ et le classème /pauvre/ désignant le trait distinctif de l'espace familier.

La seconde isotopie est d'ordre actoriel ; elle se forme dans le discours à partir de la redondance des indicateurs relatifs aux « enfants »; cette isotopie renferme d'autres groupes d'acteurs : à savoir les parents de ces enfants, les Malais et les chiens ; leur occurrence est moins fréquente que celle des enfants, mais toujours aussi significative car il existe entre les deux derniers groupes et les enfants une relation de similitude et d'appartenance.

Le regard du narrateur plane sur la plaine Kam pour s'attarder sur les enfants (accompagnés des chiens) et pour en faire un discours foudroyant qui sonne comme l'écho d'un des grands thèmes du roman: la misère. L'extrait qui nous sert de corpus conjugue ainsi deux isotopies essentielles, « spatiale » et « actorielle ». La première engobe deux valeurs sémantiques à savoir l'espace géographique et l'espace naturel ; tandis que la seconde se rattache essentiellement aux enfants et en partie aux chiens et aux Malais ; les acteurs collectifs qui peuplent la plaine.

Tableau 1: Les isotopies spatiale et actorielle du corpus

\begin{tabular}{|c|c|}
\hline Isotopie spatiale (/espace/+ /nature/) & Isotopie actorielle \\
\hline $\begin{array}{c}\text { «La plaine », " la rivière », « village », « îles vertes », « buffles » } \\
\text { " manguiers », « forêt » }\end{array}$ & " Enfants », « les Malais » et « les chiens » \\
\hline
\end{tabular}

Cette partie du roman montre précisément l'aridité de la vie indigène à travers les manières de vivre (et de mourir, voir la séquence IV) des enfants. Comme l'indique cette première séquence, ils sont partout en grand nombre et mènent une vie au gré des conditions du territoire. 
Dans cette séquence, les cinq occurrences du lexème " enfants » désignent grammaticalement et sémantiquement le «nombre pluriel » dans « beaucoup d'enfants », «les premiers enfants », « ces enfants », « les enfants » (2 occ). Le discours du narrateur traduit la régularité discursive par l'emploi systématique des pronoms personnels ou indéfinis par l'anaphorisation :

Tableau 2: Les lexèmes pour « enfants » et les anaphores SQ I

\begin{tabular}{|c|c|c|}
\hline Lexème & Nature et Fonction & Fréquence \\
\hline «enfants » & Substantif; sujet/c.o.d. & 5 occ. \\
\hline en & Pronom indéfini; compl. & 3 \\
\hline ils & Pronom personnel sujet & 2 \\
\hline tous & Pronom indéfini, apposé & 1 \\
\hline eux & Pronom personnel; compl. & 2 \\
\hline qui & Pronom relatif; sujet & 3 \\
\hline
\end{tabular}

Comme le montre le tableau ci-dessus, les « enfants » de la plaine sont 17 fois évoqués dans cette séquence pour former l'isotopie actorielle autour de la figure " enfants » qui apparaissent comme un élément de sens invariable du corpus de notre travail. Les formes discursives montrant l'omniprésence et l'abondance des enfants indigènes dans la plaine réfèrent au « fléau » par la métaphore « calamité ». Désignée comme un fait non souhaité et qui dérange, la présence des enfants est considérée comme un phénomène à la fois /humain/ et /naturel/, tandis que leur pauvreté se montre comme une condition humaine inhérente à l'environnement. Conciliant les deux valeurs " désastreuse » et " inévitable » la forme de vie des enfants s'intègre parfaitement dans l'espace principal de l'histoire, la plaine de Kam. Le discours tisse la relation d'implication et de similitude entre l'espace et ses acteurs (enfants).

L'adverbe « partout » qui se rapporte à l'isotopie spatiale désigne en même temps le mode de vie des enfants ; il est relayé dans cette séquence par les prépositions « sur », «dans », «à la pointe de », etc. pour montrer tous les lieux où l'on rencontre les enfants. Nous le considérerons également comme l'espace familier des enfants.

La séquence met en valeur trois types d'acteurs collectifs : le vocabulaire qui désigne les enfants et les chiens pullule dans cet extrait. Les Malais n'y apparaissent que vers la fin de la séquence. Les acteurs humains sont montrés par leur /faire/+/être/tandis 
que les animaux par leur /être/ ; leur description souligne leur compagnie avec les enfants. Présentés comme des acteurs principaux de la plaine de Kam, les enfants, les chiens et les Malais ont une vie soumise essentiellement à la famine et aux autres conditions défavorables. Acteurs de la plaine:

Tableau 3: La présentation des acteurs collectifs du corpus

\begin{tabular}{|l|l|}
\hline enfants & $\begin{array}{l}\text { Rêver, patauger, jouer nager, sourire (sujet des verbes) } \\
\text { vautrés dans la vase, ravis, enfermés, enduits du safran, suivis des chiens (participes) }\end{array}$ \\
\hline chiens & efflanqués, galeux, voleurs de basses-cours, maigres, coriaces (adjectif ou nom attribut) \\
\hline Malais & chasser les chiens, les manger en temps de grande famine (sujet des verbes) \\
\hline
\end{tabular}

A y regarder de près, cette différence d'ordre lexical sur la base de l'opposition « humain/animal » semble être objet d'une contigüité sur le plan sémantique. D'ailleurs, la fin du segment souligne le rapport d'implication sous forme de nécessité vitale entre les deux acteurs collectifs de la plaine : les enfants et les chiens errants sont les figures de la misère naturelle et leurs existences se valent. Les enfants et les chiens ont en commun un sort qui les réunit dans la misère et la faim ; les sèmes génériques /animal/ et /humain/ déterminent leur façon d'être dans la plaine.

Notre dernière remarque porte sur le trait figuratif inattendu concernant les enfants, à savoir leur sourire: dans ce paysage fruste, seul le sourire des enfants introduit un trait sémantique /euphorique/ puisqu'ils « souriaient mieux que personne n'a jamais souri au monde ». La constatation dévoile subrepticement le point de vue subjectif qui se rapporte plutôt à la mère ; d'ailleurs, à la fin, le lecteur apprendra qu'elle avait gardé chez elle quelques-uns de ces enfants pour les sauver de la mort. Le lien affectif de la mère pour les enfants de Kam se trouve ainsi repris. Leur beau sourire, signe d'insouciance propre à ce mode de vie est tout ce qui est /euphorique/ dans le corpus.

\section{Séquence II : « Dès le coucher du soleil... "}

Dès le coucher du soleil les enfants disparaissaient à l'intérieur des paillotes où ils s'endormaient sur les planchers de lattes de bambous, après avoir mangé leur bol de riz. Et dès le jour ils envahissaient de nouveau la plaine, toujours suivis par les chiens errants qui les attendaient toute la nuit, blottis entre les pilotis des cases, dans la boue chaude et pestilentielle de la plaine. (Duras, 1950, p. 116) 
Le lexème « enfants » désigné 4 fois (" enfants » (1) et repris par les pronoms « ils (2), les (1)») introduit une première isotopie actorielle qui s'accompagne d'une deuxième isotopie actorielle désignant « chiens » qui se présente dans 2 occurrences (« chiens » et le pronom « qui »). Comme dans la séquence précédente, les chiens sont désignés par l'adjectif épithète « errants » présentés comme leur principale qualité et qui semble métaphoriquement s'appliquer à l'acteur collectif « les enfants » de la plaine. Les deux acteurs collectifs ont en commun le trait figuratif « errants » qui réduit l'écart entre les deux isotopies « humaine vs animale » en créant une sorte de concordance thématique entre les deux acteurs.

Les enfants et les chiens sont présentés par le système pronominal d'anaphorisation. Les substantifs (« enfants/chiens») n'apparaissent qu'une fois, et sont repris ultérieurement par les anaphores. Voici comment se présentent les occurrences du couple « enfants et chiens », preuves de leur compagnie :

Tableau 4: Système anaphorique de la SQ II

\begin{tabular}{|l|c|c|}
\hline Lexèmes & Nature et Fonction & Fréquence \\
\hline « Enfants» & Sujet (nom) & 1 occ \\
\hline «lls» & sujet (pronom pers. anaphorique) & 2 \\
\hline «Les» & c.o.d. (pronom personnel anaphorique) & 1 \\
\hline «Chiens & comp. d'agent (nom) & 1 \\
\hline «Qui » & Sujet (pr. rel. anaphorique « chiens ») & 1 \\
\hline
\end{tabular}

En suivant Adam et Goldenstein pour lesquels le concept de l'isotopie s'apparente à la problématique dénotation/connotation (1976, p. 97), les isotopies actorielles « enfant » et « chiens » s'unissent dans le texte pour évoquer la similitude entre les deux acteurs collectifs en superposant les valeurs sémantiques /humaine/ et /animale/ dans le contexte géographique dessiné dans l'énoncé. Les deux isotopies ainsi corrélées constituent la concordance entre les deux univers (enfants/chiens). Ce qui peut être lu comme une caractéristique de la vie de la plaine de Kam.

L'emploi des verbes à l'imparfait « disparaissaient » et « envahissaient » indiquent le rythme constant et monotone déterminant la vie des enfants dans la plaine ; les locutions temporelles antinomiques : " dès le coucher du soleil » vs « dès le jour » prouvent cet effet de rythme de vie. La répétitivité indiquée par la locution « de nouveau » ne concerne pas seulement les enfants, mais aussi les chiens qui descendent régulièrement 
vers la plaine à côté des enfants. Ces deux acteurs se rapprochent dans la configuration thématique de « compagnie ». Leur apparition dans la plaine selon le rythme du jour et de la nuit est mise en discours sous une forme aspectuelle qui indique que le discours de l'énonciateur- observateur est investi de l'effet de sens impliquant sa présence implicite dans cet acte cognitif (Greimas et Courtés, 1993, p. 21). Leur vie commune est décrite comme un processus figuratif et temporalisé : /répétitif/ dans « de nouveau », /inchoatif/ dans « dès le jour ») et /continu/ dans « toujours ».

Tableau 5: Les actes des sujets et la temporalité dans la mise en discours

\begin{tabular}{|c|c|}
\hline $\begin{array}{l}\text { "Enfants » } \\
\text { « dès le coucher », } \\
\text { dès le jour » }\end{array}$ & $\begin{array}{l}\text { « disparaissaient », « s'endormaient », « avoir mangé » } \approx \text { /soir/+/inchoatif/ } \\
\text { « suivis de » } \approx \text { /jour/ + /inchoatif/+ /répétitif/ }\end{array}$ \\
\hline $\begin{array}{l}\text { «Chiens » } \\
\text { «toute la nuit » }\end{array}$ & $\begin{array}{l}\text { « attendaient } » ; \text { « blottis » } \approx \text { /soir/ + /continu/ } \\
\text { «toujours suivis par des chiens » } \approx / \text { jour/ + /répétitif/ }\end{array}$ \\
\hline
\end{tabular}

En examinant les verbes du paragraphe, nous voyons que les actes " disparaître », "s'endormir », et « manger » se rapportent au « soir »; ils font partie du rythme quotidien de la vie dans la plaine, tandis que " envahir » (la plaine) est une action relative au « jour ». Ainsi se dessine-t-elle la simplicité du mode de vie menée dans la plaine. Le verbe " envahir » par son noyau sémique /mouvement/+/excessif/ est un moyen d'évaluer quantitativement la manière dont les enfants errent dans la plaine. L'énoncé se rejoint sémantiquement aux deux premiers énoncés de notre corpus : «II y avait beaucoup d'enfants dans la plaine. C'était une sorte de calamité »; et formule pertinemment la façon d'être des enfants. Leur nombre excessif, leur présence non souhaitée, leur déplacement invasif dans la plaine sont mis en discours comme un phénomène incontrôlable.

Unis aux enfants par un rapport de contiguïté, les chiens apparaissent comme les acteurs secondaires de la plaine. D'un côté, leur être semble fournir un élément de définition de l'espace. L'indication figurative « blottis dans la boue chaude et pestilentielle de la plaine » renforce non seulement la misère de la plaine, mais sert à montrer en même temps l'espace géographique où vivent la Mère, Suzanne et Joseph ; en effet, c'est un terrain d'exil choisi illusoirement pour un meilleur avenir. D'ailleurs d'un bout à l'autre, le roman est dirigé par le thème de la déception de la mère planant sur la vie des enfants de plaine, sur ses propres enfants et finalement sur sa vie à elle. Et d'un autre côté, les chiens comme figures de la plaine s'unissent aux enfants en établissant un rapport de ressemblance dans le climat commun de la misère. 


\section{Séquence III : « II en était de ces enfants comme des pluies, des fruits, des inondations »}

Il en était de ces enfants comme des pluies, des fruits, des inondations. Ils arrivaient chaque année, par marée régulière, ou si on veut, par récolte ou par floraison. Chaque femme de la plaine, tant qu'elle était assez jeune pour être désirée par son mari, avait son enfant chaque année. A la saison sèche, lorsque les travaux des rizières se relâchaient, les hommes pensaient davantage à l'amour et les femmes étaient prises naturellement à cette saison-là. Et dans les mois suivants les ventres grossissaient. Ainsi, outre ceux qui en étaient déjà sortis il y avait ceux qui étaient encore dans les ventres des femmes. Cela continuait régulièrement, à un rythme végétal, comme si d'une longue et profonde respiration, chaque année le ventre de chaque femme se gonflait d'un enfant, le rejetait, pour ensuite reprendre souffle d'un autre. (Duras, 1950, p. 117)

La séquence commence par la constatation qui reprend sémantiquement les premiers énoncés du corpus ; la tournure impersonnelle « il y avait beaucoup d'enfants » (voir la séquence1) est reprise : « II en était de ces enfants...», elle permet de construire dans la narration, I'homogénéité discursive dessinant l'aspect rudimentaire de la plaine. L'énonciation vise une fois de plus les enfants : les 9 occurrences montrent la prépondérance de l'isotopie actorielle « enfants».

Tableau 6 : Système anaphorique de la SQ III

\begin{tabular}{|l|c|}
\hline Nature et Fonction & Fréquence \\
\hline Enfant(s) nom, complément & 3 occ. \\
\hline Ils (pronom) sujet & 1 \\
\hline Ceux (pronom) compl. & 2 \\
\hline Qui (pronom) sujet & 1 \\
\hline Le (pronom) c.o.d.. & 1 \\
\hline Un autre (pronom) c. du nom. & 1 \\
\hline
\end{tabular}

Cette fois-ci, le discours du narrateur n'utilise plus la métaphore (« une sorte de fléau »), mais la comparaison « il en était de ces enfants comme des pluies, des fruits, des inondations »; comparés aux éléments de la nature, le mode de vie des enfants obéit au rythme naturel régnant dans la plaine, comme le soulignent les expressions 
" par marée régulière », " par récolte », " par floraison ». La naissance des enfants est présentée de façon la plus banale comme s'il ne s'agissait que d'un objet. Le procédé comparatif retranche le sème /humain/ de l'acteur collectif « enfant » pour le doter des sèmes /naturel/ et /végétal/.

La description des enfants met en évidence les autres acteurs que sont les femmes et les hommes, parents de ces enfants. La façon dont ils naissent est décrite comme une pure réalité fruste, un fait de la nature. Le discours du narrateur le souligne par l'emploi fréquent des pronoms indéfinis, démonstratifs ou personnels, tous des anaphores pour désigner les enfants (" en », « le », « ils », « ceux», « un autre ») ; ils sont présentés en contigüité pour établir un rapport sémantique avec le « ventre des femmes » : de façon mécanique, le ventre de chaque femme se gonfle et enfante chaque année. Pour ainsi dire, ni voulus ni souhaités par leurs parents, ils naissent, vivent et meurent suivant le rythme de la nature qui domine la vie à Kam. A cela il faut ajouter la valeur des expressions temporelles répétitives comme « rythme végétal », « marée régulière », « chaque année » traduisant la naissance des enfants considérée comme un phénomène naturel propre à la plaine.

Comme nous l'avons déjà repéré dans la séquence 1, dans cette séquence aussi le nombre excessif du substantif « enfant » est repris. L'abondance établit une isotopie thématique de « quantité » pour qualifier la vie des enfants réduite à l'indifférence. La même isotopie se repère dans la séquence suivante.

\section{Séquence IV : « Jusqu'à un an environ les enfants vivaient accrochés à leur mère »}

Jusqu'à un an environ, les enfants vivaient accrochés à leur mère, dans un sac de coton ceint au ventre et aux épaules. On leur rasait la tête jusqu'à l'âge de douze ans, jusqu'à ce qu'ils soient assez grands pour s'épouiller tout seuls et ils étaient nus à peu près jusqu'à cet âge aussi. Ensuite ils se couvraient d'un pagne de cotonnade. A un an, la mère les lâchait loin d'elle et les confiait à des enfants plus grands, ne les reprenant que pour les nourrir, leur donner, de bouche à bouche, le riz préalablement mâché par elle. Lorsqu'elle faisait par hasard devant un Blanc, le Blanc détournait la tête de dégoût. Les mères en riaient. Qu'est-ce que ces dégoûts-là pouvaient bien représenter dans la plaine ? Il y avait mille ans que c'était comme ça 
qu'on faisait pour nourrir les enfants. Pour essayer plutôt d'en sauver quelques-uns de la mort. Car il en mourait tellement que la boue de la plaine contenait bien plus d'enfants morts qu'il y en avait eu qui avaient eu le temps de chanter sur les buffles. II en mourait tellement qu'on ne les pleurait plus et que depuis longtemps déjà on ne leur faisait pas de sépulture. Simplement, en rentrant du travail, le père creusait un petit trou devant la case et il y couchait son enfant mort. Les enfants retournaient simplement à la terre comme les mangues sauvages des hauteurs, comme les petits singes de l'embouchure du rac. Ils mouraient surtout du choléra que donne la mangue verte, mais personne dans la plaine ne semblait le savoir. Chaque année, à la saison des mangues, on en voyait, perchés sur les branches, ou sous arbre, qui attendaient, affamés, et les jours qui suivaient, il en mourait en plus grand nombre. Et d'autres, l'année d'après, prenaient la place de ceux-ci, sur ces mêmes manguiers et ils mouraient à leur tour car l'impatience des enfants affamés devant les mangues vertes est éternelle. D'autres se noyaient dans le rac. D'autres encore mouraient d'insolation ou devenaient aveugles. D'autres s'emplissaient des mêmes vers que les chiens errants et mouraient étouffés. (Duras, 1950, p. 117-118)

Le paragraphe ne contient qu'un seul temps grammatical : l'imparfait que renforcent les indications temporelles « mille ans » et « chaque année » pour montrer le mouvement continu de naissance des enfants et de leur mort précoce dans la plaine. Cette séquence se distingue de la précédente par l'évocation de la mort des enfants, qui elle aussi se décline sur le mode $d^{\prime}$ '« indifférence » ou d' « insignifiance ». Pour ainsi dire, le rythme naturel de la vie des enfants se révèle validé par la mort.

Le système d'anaphores désignant enfants les reprend 5 fois. Les anaphores sont au nombre de 25 et ensemble dessinent l'isotopie actorielle de cette séquence.

Tableau 7: Système anaphorique de la SQ IV

\begin{tabular}{|l|c|c|}
\hline Enfants /enfant & Nom/ sujet c.o.d., c.o.i. & 7 occ. \\
\hline Ils & Pronom personnel sujet & 5 \\
\hline Leur/les & Pronom personnel/c.o.d., c.o.i. & 5 \\
\hline D'autres & Pronom indéfini/sujet & 1 \\
\hline Ceux-ci & Pronom démonstratif/c.du nom & 5 \\
\hline En & Pronom indéfini/c.partitif & 4 \\
\hline
\end{tabular}


Comme le montre la liste des déterminants anaphoriques remplaçant le lexème « enfants », les enfants continuent d'être les principaux acteurs de ce passage essentiel, d'une part, et d'autre part, ils rendent compte des conditions naturelles de l'espace principal de l'intrigue que nous avons dénommé plus haut comme l'espace familier des trois protagonistes du récit.

Dessinant le cadre topologique de l'histoire narrée, la plaine de Kam apparaît impensable sans le recours figuratif aux acteurs « enfants ». Ceux-ci sont présentés d'abord comme sujet : la première occurrence des enfants a pour verbe « naître », or les autres occurrences montrent le cheminement des mêmes enfants vers la vie et puis vers la mort. Les verbes indiquent les étapes de leur vie : être nus, tout petits, ensuite vêtus d'un pagne, puis assez grands pour se dépouiller tout seuls, nourris puis lâchés par leur mère dans la plaine, et finalement « morts » tout jeunes. Les indications grammaticales (sujets ou complément) et les verbes qui s'y rattachent connotent la quantification (ils sont en grand nombre) et c'est ainsi que la narration rend compte de la vie des enfants de la plaine.

Les 13 occurrences du sème nucléaire /mort/ contenu dans les lexèmes ci-dessous manifestent au niveau profond la présence de l'isotopie de la « mort » :

Tableau 8: L'isotopie de la « mort »

\begin{tabular}{|l|c|}
\hline (arracher à) la mort & 1 occ \\
\hline enfant(s) morts(s) & 2 \\
\hline il en mourait (sujet impersonnel) / ils mouraient: & 7 \\
+ se noyaient /s'emplissaient des vers & +2 \\
\hline sépulture & 1 \\
\hline
\end{tabular}

La séquence use des anaphores « en » de l'indéfini « il y en avait tellement », « il en mourait tellement » (2 occ.), pour montrer la quantification qui s'installe dans ce discours comme un élément évaluateur. Le nombre excessif des enfants explique en même temps la façon dont ils meurent. Leur mort est ordinaire; à tel point que les parents finissent par les enterrer dans la boue de la plaine. Devenu habituelle, la mort est objet d'indifférence. Le monde des Blancs et celui des indigènes s'opposent donc non seulement dans leur façon de nourrir les enfants, mais aussi dans leur manière de considérer la mort. Le « trop plein » mène à l'indifférence par rapport à la vie et à la mort : « il en mourait tellement qu'on ne les pleurait plus » (voir le paragraphe ci-dessus).

Dans cette séquence, la quantification doit être considérée comme un effet du discours qui prend à sa charge l'isotopie affective de l'« indifférence » qui se rapporte 
non seulement aux modes de vie des enfants, mais aussi à l'état des parents habitués à la perte de leurs enfants. La pauvreté empêche la sensibilisation du sujet collectif « parents » qui laissent les enfants naître, grandir et mourir dans une sorte d'indifférence, obéissant au seul rythme de la nature. La comparaison est claire et établit la contigüité entre les sèmes /humain/, /animal/ et /végétal/ qui caractérisent l'être des enfants: «les enfants retournent à la terre comme les mangues sauvages et les petits singes ».

La mort se présente ainsi sous toutes ses formes : ils meurent de choléra, noyés dans le rac, d'insolation ou étouffés par les vers. Aux enfants, la plaine ne donne rien, sauf les mangues vertes qui provoquent le choléra, les vers qui les étouffent, le soleil violent qui les tue ou les rend aveugles. Sans nourritures, puis sans sépultures, leur vie est décidée dès la naissance pour durer très peu et pour peupler la plaine pour un certain temps. De la même manière que la naissance est un incident ordinaire, la mort est considérée comme un incident ordinaire dans la plaine.

\section{Séquence V : « Et il fallait bien qu’il en meure »}

Et il fallait bien qu'il en meure. La pleine était étroite et la mer ne reculerait pas avant des siècles, contrairement à ce qu'espérait toujours la mère. Chaque année, la marée qui montait plus ou moins loin, brûlait en tout cas une partie des récoltes et, son mal fait, se retirait. Mais qu'elle montât plus ou moins loin, les enfants, eux, naissaient toujours avec acharnement. Il fallait bien qu'il en meure. Car si pendant quelques années seulement, les enfants de la plaine avaient cessé de mourir, la plaine en eût été point infestée que sans doute, faute de pouvoir les nourrir, on les aurait donnés aux chiens, ou peut-être les aurait-on exposés aux abords de la forêt, mais même alors, qui sait, les tigres eux-mêmes auraient peut-être fini par ne plus en vouloir. Il en mourait donc et de toutes les façons, et il en naissait toujours. Mais la plaine ne donnait toujours que ce qu'elle pouvait de riz, de poisson, de mangues, et la forêt, ce qu'elle pouvait aussi de maïs, de sangliers, de poivre. Et les bouches roses des enfants étaient toujours des bouches en plus, ouvertes sur leur faim.

La mère en avait toujours eu un ou deux chez elle pendant les premières années de son séjour dans la plaine. Mais maintenant elle en était un peu dégoûtée. (Duras, 1950, p. 118-119) 
Comme dans tout l'extrait, cette petite séquence aussi se caractérise par l'utilisation abondante des pronoms désignant l'acteur collectif « enfants ». Deux fois il est désigné par le substantif, pour laisser sa place dans le discours aux pronoms définis et indéfinis comme anaphores. Employé comme complément du nom, complément déterminatif, le pronom « en » a pour effet de sens « quantité indéterminée ». Le pronom personnel « les » employé trois fois (donc moins de fois par rapport à « en ») est complément d'objet direct pour les verbes « nourrir », « les donner aux chiens » « les aurait exposés ». L'énumération des verbes laisse voir une logique qui oppose « vie / mort » ; « vie » pour nourrir et « mort » pour se débarrasser d'eux. Le système d'anaphores (surtout la récurrence des « en ») montre l'être des enfants plutôt comme « impersonnel » et « objet » comme l'indique le contenu de la catégorie grammaticale de « en/les »

Tableau 9: Système anaphorique de la SQ V

\begin{tabular}{|l|c|}
\hline Les enfants (de la plaine) & 2 occ \\
\hline (les bouches des) enfants c.du nom & 1 \\
\hline En pronom indéf / impersonnel /quantifié & 7 \\
\hline Eux pronom pers. apposition & 1 \\
\hline Les pronom pers. c.o.d. & 3 \\
\hline
\end{tabular}

La dernière séquence du corpus se présente comme une argumentation : la mort des enfants s'avère comme une «nécessité » devant le rythme de naissance acharné et la pénurie dans la pleine. S'ils ne meurent pas « pendant quelques années seulement », la plaine en serait tellement remplie qu'il faudrait s'en débarrasser en les donnant aux animaux sans être sûr s'ils les mangeraient. L'oscillation entre la naissance et la mort est une condition intrinsèque de la vie de la plaine. Le rythme de la vie d'un enfant est conditionnée par la famine.

La grammaire de la restriction dans « pendant quelques années seulement », « faute de pouvoir les nourrir », « ne donnait toujours que ce qu'elle pouvait... » (2 occ.) et celle d'impératif et de contrainte dans « il fallait qu'il en meure » relayé par « il en mourait donc » installent dans le discours l'isotopie de « pénurie » et justifie la mort nécessaire des enfants. L'isotopie thématique « pénurie » détermine le mode de vie des indigènes soumis à l'insuffisance foncière des vivres. Ainsi décrite, toute la vie dans la plaine semble basculer entre la « profusion » des enfants et l' « insuffisance » des moyens de vie. L'opposition sémantique " profusion » vs « restriction » s'inscrit sur l'axe de la quantification que nous avons observée comme élément discursif de tout le passage étudié. La profusion concerne le nombre d'enfants nés et le, nombre d'enfants morts ; tandis que la restriction se rattache à la nourriture. 


\section{De l'énoncé à l'énonciation}

Qu'ils soient grammaticaux ou figuratifs, tous les éléments du discours se considèrent dans l'analyse comme relevant du niveau de l'énoncé. Construisant le texte comme un énoncé suivi, ces mêmes unités installent au niveau profond les isotopies qui sont les plans homogènes de la signification du texte. Pour repérer le vaste système de désignation, nous avons analysé les données énoncives du texte tels que les substantifs, les pronoms anaphores, les verbes employés. Conformément à la théorie de l'énonciation, nous considérons les faits énoncifs comme résultant de l'acte de l'énonciation qui installe dans l'énoncé, les points de vue d'un sujet observateur, focalisateur, informateur etc.

Il importe alors de s'appuyer sur les faits du discours tel qu'il est construit par le sujet de l'énonciation. II va sans dire que les répétitions anaphoriques et la récurrence des éléments indéfinis/impersonnels dénotent un discours mettant l'accent sur l'indifférence des habitants de la plaine à la tragédie des enfants. La naissance comme la mort sont considérées comme des faits ordinaires.

Les tournures présentatives ci- dessous répétés 10 fois dans l'extrait analysé font également penser aux traits sémiques /inanité/+/impersonnel/ :

Tableau 10 : Les présentatifs impersonnels

\begin{tabular}{|l|c|}
\hline «il y avait beaucoup d'enfants» & 1 occ \\
\hline «il en était de ces enfants » & 3 \\
\hline «il y en avait qui »... & 2 \\
\hline «il en mourait...» & 4 \\
\hline
\end{tabular}

L'effet de quantification se déploie sur les lexèmes grammaticaux et sur le système des anaphores pour collaborer à la mise en œuvre des isotopies thématiques de « misère » et de « pénurie » étroitement liées à celle de « mort ».

Pour le repérage des isotopies nous avons recouru à la fréquence des mots grammaticaux, des déterminants, des pronoms et des anaphores. Comme le montre l'analyse, les catégories grammaticales d'indéfini/impersonnel et de quantité œuvrent dans le récit pour déboucher sur les isotopies thématiques : les catégories grammaticales isotopantes tentent d'homogénéiser les couches polysémiques de la structure profonde. 
Les occurrences du substantif « enfants » (au total 19 occurrences) déterminent l'isotopie actorielle /collective/, ce qui fait qu'au niveau profond, l'identité collective des enfants est essentiellement dépourvue du trait sémique nucléaire /individuel/.

La quantification, comme procédé énonciatif, présente les enfants à un grand nombre indéfini. Comme nous laisse entendre le texte de Duras, ils sont là depuis que la plaine existe et sont les éléments impertinents de cette géographie. Nés et morts irrémédiablement dans la pauvreté et la misère, les enfants tracent la configuration thématique de la fusion entre " vie » et «mort ». Le texte semble effacer les limites entre les deux deixis. Toutefois les différents traits figuratifs des enfants nous permettent de saisir l'organisation sémantique de l'isotopie « vie/mort » sous la forme d'un carré sémiotique qui illustre les traits sémiques inhérents à leur être :

\section{Schéma 1 : Le carré sémiotique de l'isotopie " vie/mort »}

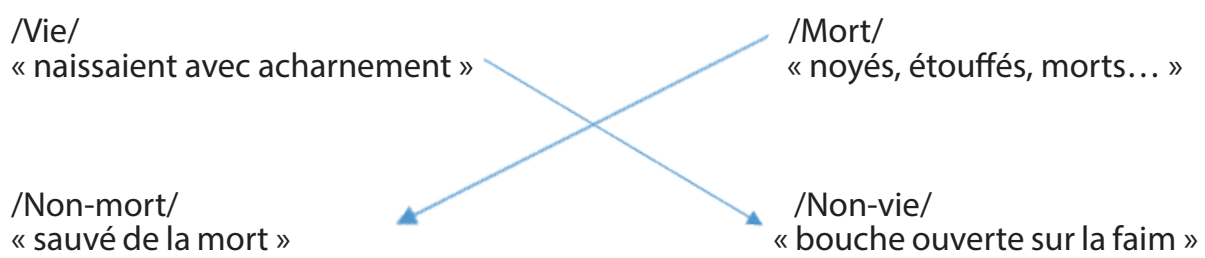

Pour finir, la manière dont les enfants meurent montre la constance des conditions misérables de la plaine. Le manque d'espoir, l'effacement de l'opposition sémantique entre « vie et mort » accentue le drame des enfants. La pauvreté apparaît comme une raison de l'indifférence à l'égard du nombre d'enfants vivants et morts. L'indifférence des indigents est reflétée par l'abondance des éléments anaphoriques connotant l'impersonnalité, l'anonymat, l'inanité au niveau de la surface du texte.

En fin de compte, il est clair que la plaine de Kam, qui apparaît comme un élément important est synonyme de la misère, de la pauvreté et de la mesquinerie, symbolise en quelque sorte l'échec et l'indifférence à l'égard de la vie et de la mort. Comme le montre notre analyse, la plaine est thématiquement connectée au mode de vie des enfants qui la peuplent. Entre l'acteur collectif « indigènes+ enfants » et cet espace anonyme et aride existe un lien métonymique ; ceux-ci sont sans nom, sans personnalité, sans trait particulier dans un territoire qui ne leur rapporte rien que la maladie et la mort. 


\section{Bilan de l'analyse et conclusion}

Le corpus de notre analyse illustre les axes thématique et figurative du roman et apparaît comme un exemple significatif du discours romanesque qui met l'accent sur les personnages anonymes de l'histoire racontée. Misé par le point de vue observateur, les enfants apparaissent comme une figure anonyme et collective dont le destin tragique fait penser à celui des petits colons du pays.

Dans le texte, les enfants sont comparés aux animaux et aux plantes qui poussent dans la plaine d'eux mêmes. Leur vie et leur survie, comme la mort, est un fait ordinaire, à tel point que leur naissance et leur mort sont invariablement soumis à l'indifférence de leur famille. Les procédés comparatifs, l'emploi des verbes et des adjectifs illustrent le point de vue opté dans la narration pour décrire les conditions minables de la plaine comme espace de vie des indigènes.

La mise en discours des procédés stylistiques rapprochant les «enfants » et les «chiens » dans le cataclysme, la vie et la mort dans l'indifférence, se trouve véhiculée de façon particulière par un réseau d'anaphores employées surtout pour « enfants ». L'analyse de la fréquence des « indéfinis » et de leur contenu sémique a montré le rapprochement des catégories sémantiques «/animale/humaine», « vivant/mort», « sens/insignifiance » au sein de la configuration thématique « enfants ».

Ainsi, le grand nombre de pronoms indéfinis à valeur d'indéfini se répercute sur l'isotopie thématique « inanité » définie comme « caractère de ce qui est vide, sans contenu réel, qui ne présente aucun intérêt pour le cœur ou pour l'esprit » pour décrire l'être des enfants de la plaine. Tout le système anaphorique du corpus souligne le caractère nonsignifiant de la vie des enfants en les réduisant dans le discours à l'état « inanimé ».

Par conséquent, la ligne de démarcation sémantique entre " animé/ inanimé », entre « humain/animal » et «sensé/insignifiant » s'efface dans le texte. Nous pouvons ainsi considérer le jeu d'inversion et/ou de rapprochement des valeurs sémantiques des figures comme « enfants, chiens, vie, mort, fléau » comme un fait de style en jouant sur l'ambigüité référentielle de ces figures par rapport à leur contenu figuratif et thématique.

Comme dans cet extrait analysé, le roman de Duras est en soi-même une dénonciation des conditions indignes infligées non seulement à certains Français expatriés dans les 
colonies, mais aussi aux peuples de ces pays. La nouvelle vie que la Mère souhaitait construire ici est devenue un parfait exemple d'échec (dettes, pauvreté, ennui, détresse, désespoir). Or comme nous le montre la description de la vie dans la plaine, dans ces confins, il n'y a pas de moyen efficace pour réorganiser une vie prospère. L'extrait souligne, à travers d'images fortes, le vain acharnement de la Mère pour construire un barrage contre le Pacifique.

Rien ne peut pousser sur ces terres comme nous le montrent les enfants condamnés aux maladies et à la mort. La déception de la Mère sombrée dans la détresse est homologuée au destin des enfants de la plaine. Au départ, elle avait gardé des enfants auprès d'elle pour en sauver au moins quelques-uns de la mort. Toujours sans succès.

Comme nous le rappellent Adam et Goldenstein, « le texte littéraire n’est pas centré sur le réel dénotatif, mais sur la mise en action du potentiel connotatif des signes » $(1976, p$. 94). Le destin précaire des enfants et leur vie de «fléau » se rejoint implicitement à l'échec des barrages écroulés, qui donne le titre au roman. L'analyse a su montrer la présence d'un rapport sémantique et sémiologique entre ce qui est narré et ce qui relève de l'univers du roman par un subtil jeu de l'énonciation brouillant les effets connotatifs et symboliques au niveau énoncif. Par un jeu d'implication sémantique entre la vie des protagonistes et le destin des personnages secondaires (les enfants de la plaine) l'énonciation rend visible et sensible un univers de dépérissement et de perte propre au récit de Duras.

Évaluation : Évaluation anonyme par des pairs extérieurs.

Conflit d'intérêts : L'auteure n'a aucun conflit d'intérêts à déclarer.

Subvention : L'auteure n'a reçu aucun soutien financier pour ce travail.

Peer-review: Externally peer-reviewed.

Conflict of Interest: The author has no conflict of interest to declare.

Grant Support: The author declared that this study has received no financial support.

\section{Bibliographie}

Adam, J. -M. et Goldenstein, P. (1976). Linguistique et discours littéraire, théorie et pratique du texte. Paris : Larousse Université.

Bertrand, D. (2000). Précis de sémiotique littéraire. Paris : Nathan.

Duras, M. (1950). Un barrage contre le Pacifique. Paris : Gallimard (Folio).

Fontanille, J. (1989). Les Espaces subjectifs. Introduction à la sémiotique de l'observateur. Paris : Hachette.

Greimas, A.J. (1976). Maupassant. La sémiotique du texte : exercices pratiques. Paris : Seuil.

Greimas, A.J. et Courtés, J. (1993). Sémiotique. Dictionnaire raisonné de la théorie du langage. Paris : Hachette.

Rastier, F. (1989). Sens et Textualité. Paris : Hachette. 
